ÉGYPTE monde arabe

\section{Égypte/Monde arabe}

$10 \mid 1992$

Partage de l'eau dans le monde arabe

\title{
Rachid Ghannuchi : Islam, nationalisme et islamisme (entretien)
}

\section{François Burgat}

\section{(2) OpenEdition}

\section{Journals}

Édition électronique

URL : https://journals.openedition.org/ema/1420

DOI : 10.4000/ema. 1420

ISSN : 2090-7273

\section{Éditeur}

CEDEJ - Centre d'études et de documentation économiques juridiques et sociales

\section{Édition imprimée}

Date de publication : 30 juin 1992

Pagination : 109-122

ISSN : 1110-5097

\section{Référence électronique}

François Burgat, « Rachid Ghannuchi : Islam, nationalisme et islamisme (entretien) », Égypte/Monde arabe [En ligne], 10 | 1992, mis en ligne le 08 juillet 2008, consulté le 07 juillet 2022. URL : http:// journals.openedition.org/ema/1420 ; DOI : https://doi.org/10.4000/ema.1420

Ce document a été généré automatiquement le 7 juillet 2022.

Tous droits réservés 


\title{
Rachid Ghannuchi : Islam, nationalisme et islamisme (entretien)
}

\author{
François Burgat
}

\section{NOTE DE L'ÉDITEUR}

Entretien recueilli (à Londres au mois de février 1992) et traduit de l'arabe par François Burgat

\section{Présentation}

1 La publication de cet entretien avec Rachid Ghannûchi, leader en exil du Parti islamiste tunisien Al-Nahda, dont l'influence s'étend bien au-delà du terroir maghrébin, s'inscrit dans le prolongement des rencontres avec 'Adil Husayn, rédacteur en chef de l'hebdomadaire Al-Cha'b et membre dirigeant du Parti du Travail, et avec l'historienjuriste Tariq al-Bichri, publiées respectivement dans les numéros 5 et 7 d'Égypte/Monde Arabe.

2 Ces documents font partie d'une série d'interviews d'intellectuels du courant islamiste, égyptiens, maghrébins mais aussi jordaniens, soudanais et yéménites, en cours de collecte au CEDEJ dans le cadre d'une recherche sur les conditions d'articulation des formations islamistes à la scène politique légitime ${ }^{1}$. La présentation de l'itinéraire des cadres intellectuels et/ou politiques $\mathrm{du}$ mouvement islamiste relève d'une double préoccupation méthodologique :

3 1. L'étude des itinéraires individuels d'adhésion à la thématique islamiste et des mécanismes de ces "conversions ", tels qu'ils sont verbalisés par les intéressés, vise d'abord à nourrir une hypothèse de recherche énoncée de longue date (in L'Islamisme au Maghreb, cité). Cette hypothèse fait de la dimension nationaliste de la thématique 
islamiste sa composante fondatrice et structurante, et propose d'organiser autour d'elle la lecture des différents facteurs adjuvants, économiques ou politiques, qui en spécifient, dans le temps et dans l'espace, les diverses expressions sociales et nationales.

Le contexte de l'itinéraire de Rachid Ghannûchi vers l'islamisme comporte à la fois une première étape marquée de spécificité tunisienne et un second temps - celui précisément de la transition du nationalisme laïque à l'islamisme - plus proche du modèle quasi universel qui conduit aujourd'hui bon nombre d'intellectuels arabes à adhérer aux représentations islamistes. La laïcisation et la francisation linguistique volontaristes imposées par Habib Bourguiba après l'indépendance de 1956 s'opèrent, en Tunisie comme ailleurs, à contre-courant des représentations culturelles dominantes chez une large majorité de la population, élites comprises. La spécificité tunisienne tient ici à ce que le facteur linguistique est plus particulièrement explicite et qu'il joue dans le temps de manière plus précoce : c'est dès l'indépendance que le choc d'une politique culturelle délibérément francophone va créer cette "armée des vaincus de Bourguiba » qu'évoque Rachid Ghannûchi.

Cette situation n'a pas d'équivalent exact dans la situation algérienne : la déculturation linguistique des élites y a été de toute façon bien plus profonde et, à l'opposé, le déclassement relatif des "arabophones " n'a joué dans la mobilisation islamiste son rôle potentialisateur, maintes fois souligné, que bien plus tardivement, une fois apparus sur le marché du travail les premiers produits de la politique de ré-arabisation de l'enseignement. Le Maroc, où la fin d'un protectorat par ailleurs plus bref a vu le régime renouer avec l'univers symbolique du système politique précolonial, n'a pas connu de rupture - entre les énoncés du régime et la culture religieuse populaire comparée à celle qui a caractérisé la Tunisie bourguibienne. Au Machreq (où Ghannûchi s'expatrie à la recherche d'une arabité perdue), le facteur linguistique a manifestement été moins déterminant, le recul, très réel au demeurant, de l'arabe n'ayant jamais pris, ni avant ni après l'indépendance, la même ampleur qu'au Maghreb.

6 Tel que le verbalise le leader en exil du Parti Al-Nahda, le mécanisme de sa prise de distance à l'égard de l'univers du nationalisme arabe laïque (en l'occurrence du nassérisme, qui a constitué le réceptacle politique de ses premières retrouvailles avec l'arabité) jalonne par contre des voies proches de I'« universel » islamiste, au moins pour ceux des militants politiques de cette génération (c'est-à-dire, au Maghreb, à peu près tous et au Machreq un très grand nombre) qui ont adhéré un temps à un univers autre que celui de la pensée religieuse. Après en avoir constitué le repoussoir privilégié, le langage du nationalisme laïque est peu à peu associé à l'univers symbolique occidental et c'est alors la culture religieuse qui, irrésistiblement, apparaît alors comme la seule capable de satisfaire la demande identitaire.

7 2. La diffusion de libres expressions d'acteurs du courant islamiste procède également du parti pris de faire entendre celui des versants de leur « double discours » supposé, qui ne parvient sans doute que trop rarement aux oreilles occidentales, notamment francophones. Les médias, et parfois (plus rarement il est vrai) les chercheurs ne prennent pas toujours le temps d'instaurer avec les interlocuteurs islamistes la relation de confiance qui permet de dépasser le premier stade réactionnel, et aux rencontres de sortir ainsi du cadre étroit des interrogations en forme de réquisitoire.

8 C'est pourtant, nous semble-t-il, dans ce niveau d'expression-là de la thématique islamiste qu'il faut plus que jamais, aujourd'hui, chercher les clefs de sa 
compréhension. Le propos n'est pas tant de démontrer qu'il existerait de "bons » islamistes - qui viendraient en quelque sorte compléter un paysage composite - que de mettre en évidence que c'est chez les intellectuels qu'il faut chercher la clef de lecture d'une dynamique idéologique et politique, plus certainement que dans les expressions qu'en fournissent, lorsqu'ils se l'approprient, les compartiments les plus frustes de son terroir humain, trop souvent et trop exclusivement sollicités par les feux de notre actualité.

\section{L'entretien}

« Mille neuf cent soixante-six, le 15 juin. C'est au cours de cette nuit-là que j'ai pris la décision finale de passer de l'univers du nationalisme arabe et nassériste à celui de l'Islam » «II n'est simple pour personne de reconstruire avec précision l'itinéraire exact du passage d'un univers intellectuel et idéologique à un autre et toutes les transformations - en termes de relations humaines notamment - que cela a impliqué.

10 Car c'est bien ce que ce changement, dans sa phase initiale surtout, impliquait: le passage d'un monde à un autre, d'une pensée, d'une idéologie, d'un système de relations, d'un itinéraire, à un autre univers, à de nouvelles idées, à de nouvelles valeurs et à de nouvelles relations. Cela équivalait à une brutale métamorphose.

11 Je peux bien sûr retracer le contexte événementiel d'un tel bouleversement. Autre chose est de reconstruire l'enchaînement exact des faits et surtout de prendre à leur égard la distance de celui qui visionne un film. L'essentiel se joue à l'intérieur de l'âme, de manière peut-être même inconsciente. Comment tout cela s'est-il produit? Comment en suis-je arrivé là ? Une chose est certaine, je puis dater très précisément ce changement : la nuit du 15 juin 1966.

12 C'est au cours de cette nuit-là que j'ai pris la décision finale de passer de l'univers du nationalisme arabe et nassériste à celui de l'islam. Je venais de prendre brutalement conscience que ni ce qui j'étais ni ce que je vivais n'avait quoi que soit à voir avec l'islam. Cette découvert a pris [...] pour moi des allures de catastrophe : je réalisais que je n'étais pas musulman, que j'étais étranger à l'islam.

\section{L'armée des vaincus de Bourguiba}

13 J'avais pourtant été élevé dans un environnement très religieux. Mon père était de ceux qui avaient appris tout le Coran. Il faisait les prières en famille. Il répandait de l'encens dans la maison, comme le font les gens très religieux. Ce n'était pas un "professionnel de la religion", c'était un simple paysan, mais c'est lui qui assurait l'enseignement coranique et religieux à tout le village [...].

Ensuite, j'ai reçu une éducation religieuse à l'université de la Zitouna. Cet enseignement, toutefois, n'a pas servi en quoi que ce soit à enraciner en moi la valeur de l'islam, ni permis de prendre conscience de sa capacité à régir les existences. Il faut, pour le comprendre, préciser qu'avec le retour d'exil de Bourguiba, la Tunisie vivait alors une période de bouleversement. 

constituait plutôt une victoire sur la civilisation arabo-islamique en Tunisie. Bourguiba est entré en vainqueur et, comme les envahisseurs étrangers, il a pris le pouvoir. Puis il s'est mis à frapper les institutions religieuses, ces institutions qui étaient la vie même de la Tunisie. À cette époque, tout tournait autour de l'institution de la Zitouna: l'artisanat traditionnel, la littérature tunisienne, la pensée tout entière. Jusqu'à un certain point, toute la Tunisie est le produit de la Zitouna. Alors, le coup violent porté à ces institutions a touché la structure sociale, économique et culturelle de la Tunisie tout entière. Pour tous ceux qui avaient été élevés dans la culture arabe et musulmane, c'est-à-dire pour la majorité des intellectuels tunisiens - qui sortaient de ces institutions d'enseignement rattachées à la Zitouna - arabité et islam étaient liés. Ils donnaient une grande importance à cette langue et à cette littérature arabes qui enracinaient l'appartenance arabo-islamique en Tunisie, qui y insufflait l'esprit de résistance à l'invasion étrangère [...] et servait en quelque sorte, face à l'Europe, de bouclier.

L'attaque contre les institutions religieuses fut l'une des premières décisions postérieures à l'indépendance. Ma génération s'est ainsi sentie mise en situation d'extranéité ; elle est devenue sujette à une profonde aliénation, victime d'une sorte de bannissement. Nous avions constitué un bastion arabe et islamique et le pays, à l'opposé, s'occidentalisait : l'administration, la culture, l'université, l'enseignement, les arts, les lettres..., tout s'occidentalisait. Les gens ont commencé à se demander s'ils étaient encore vraiment dans leur pays, dans leur patrie. Il fallait en effet, comme disait Bourguiba, "tout faire pour rattraper le cortège de la civilisation», pour s'intégrer, se fondre dans l'environnement occidental. À cette génération, on ne demandait donc ni plus ni moins que d'ôter ses vêtements, de s'arracher la peau en quelque sorte, pour s'intégrer à ce monde nouveau dans lequel Bourguiba jetait toute la Tunisie. Même ceux qui avaient étudié à la Zitouna, estimant qu'il n'y avait pas d'avenir pour la culture arabo-islamique, se mirent alors à envoyer leurs enfants au Lycée Carnot ou Sadiqi ou dans les établissements étrangers.

Pour les diplômés de ces institutions, le résultat de tout cela a pris la forme de troubles, de complexes d'infériorité, de sentiments d'aliénation. Il ne leur restait qu'à se replier, se recroqueviller sur eux-mêmes. Et à cuver leur rancoeur contre cette nouvelle occupation. Non plus celle des Français cette fois, mais celle des « fils tunisiens de la France ». Et celle-ci était plus brutale encore que celle-là. Les Français au moins étaient clairement identifiés comme tels [...]. Pour la génération nourrie de culture arabomusulmane qui fréquentait la Zitouna et les institutions traditionnelles, le processus d'occidentalisation de la Tunisie a donc été vécu comme une violence. Cette génération a été réprimée, elle a été victime d'un traumatisme. Or cette génération représentait la majorité. À l'indépendance, ceux qui fréquentaient les établissements dépendants de la Zitouna étaient entre 25 et 27.000 , et ceux qui étudiaient dans les établissements secondaires créés sous occupation française entre 4.500 et 5.000. C'est donc la majorité qui s'est sentie marginalisée par la minorité. C'est une minorité agissante qui a été capable de marginaliser la majorité car elle avait la capacité de comprendre l'Occident, de comprendre l'étranger et de communiquer avec le nouvel ordre international. La majorité n'avait pas ce savoir-là. Elle s'est donc fait marginaliser intellectuellement. Il en a été de même pour tout ce substrat socio-économique qui dépendait plus ou moins de la Zitouna, le secteur de l'artisanat traditionnel qui l'entourait, mais aussi le secteur

Égypte/Monde arabe, $10 \mid 1992$ 
des Waqfs, les biens Habous qui représentaient une part importante de la propriété foncière. Un quart environ de la propriété agricole en Tunisie était rattaché aux Waqfs et tout particulièrement à la Zitouna [...]

18 Le fait de frapper la Zitouna, le fait de frapper les institutions économiques qui la finançaient équivalait donc à détruire la société. Cela aboutissait aussi à mettre la génération élevée au sein de ces institutions dans la situation d'une armée vaincue et défaite. Il ne lui restait plus qu'à se fondre dans la nouvelle armée, mais il lui fallait, pour ce faire, sortir de sa propre peau. Ou bien se replier, se recroqueviller sur ellemême. Bon nombre des gens de ma génération étaient attirés par l'Orient. Si le nouvel ordre international que représentait Bourguiba en Tunisie tirait, et ses représentants avec lui, sa légitimité de l'Occident, ceux qui refusaient la violence de ce changement forcé tournaient leurs regards vers l'Orient, vers les sources de la pensée islamique et arabe où ils cherchaient appui. Il venait de surcroît de se produire dans cet Orient de multiples bouleversements dont le moins important n'était pas le coup d'État, que l'on appela par la suite la Révolution de Gamal Abdel Nasser.

Cette révolution exaltait les idées arabistes, l'unité arabe et la résistance à l'ordre international. Les Zitouniens avaient donc tout particulièrement vocation à être nasséristes, ils étaient nasséristes "par nature ». Sans s'arrêter à la dimension laïque ou au fait que c'était une autre expression du nationalisme, ils trouvaient dans le nassérisme l'appui moral qu'ils recherchaient: au Maghreb, la distinction entre l'arabité et à l'islam n'a jamais eu cours. La Zitouna prônait indistinctement un islam arabe ou une arabité musulmane. En Afrique du Nord, islamisation ou arabisation allaient de pair. Toute agression étrangère s'en prenait d'ailleurs, - en Tunisie comme en Algérie - à l'arabité et à l'islam à la fois. L'appartenance arabe est ensuite d'autant plus étroitement imbriquée avec la religion qu'il n'existe pas au Maghreb de minorités religieuses et que cette région n'était pas arabe avant l'islam. L'Afrique du Nord est entrée en contact avec l'arabité et avec l'islam en même temps. L'arabité n'a donc pas d'identité culturelle autre que musulmane, alors que les régions du Machreq se sont arabisées avant de devenir musulmanes. Il y avait des tribus arabes qui étaient chrétiennes et le sont demeurées et d'autres qui se sont islamisées. L'arabité y est donc perçue comme un tout antérieur à l'islam et on craint parfois, en faisant usage de la référence islamique, d'établir une sorte de ségrégation, problème qui ne se pose pas en Afrique du Nord.

20 J'ai déjà dit que, dans ce contexte, la vie me pesait : je ressentais une sorte d'aliénation. Je passais des heures à l'extérieur de mon village, souvent dans les jardins, à lire des nouvelles et des romans. La radio? Oui, j'écoutais Sawt al-Arab, les radios du Machreq surtout. Je lisais aussi Naguib Mahfouz. Tawfik al-Hakim. Après ma sortie de la Zitouna, je suis resté un an et demi à travailler comme instituteur. Mais cette vie ne me convenait pas. J'étais habité par ce sentiment d'aliénation (ighitirâb) qui était celui de toute ma génération. Nous éprouvions pour les sources de l'islam et de l'arabité au Machreq une sorte d'attirance nostalgique. Je suis donc parti en Égypte. C'était en 1964.

\section{... à la recherche de l'arabité perdue}

21 La découverte de l'Égypte? Mon point de vue n'a pas vraiment changé. Bien sûr, à mon arrivée au Caire, le rêve qu'avait nourri mon refus du bourguibisme, la littérature égyptienne, les chansons d'Oum Kalthoum, d'Abdel Wahab et de Farid al-Atrache, ou 
encore Radio-Égypte et les discours de Nasser, cette image lumineuse s'est quelque peu dissipée. J'ai d'abord eu d'énormes difficultés à obtenir une inscription à l'Université. Nous étions une quarantaine dans le même cas, à être venus d'Afrique du Nord. Nous ne faisions pas partie d'un groupe envoyé officiellement. On s'était seulement rencontrés ici. On était venus par voie de terre, à travers la Libye. Chacun était à la recherche de lui-méme [...]. L'État de Bourguiba n'appréciait pas que les étudiants tunisiens aillent fréquenter les universités du Machreq de peur qu'ils ne soient atteints de "folie " nassériste, ba'thiste ou arabiste. Quelques contingents officiels avaient pourtant été envoyés en Syrie. Mais notre groupe n'avait pas eu cette chance. Il a été chassé... ! : tous rescapés de la Zitouna [...], certains "survivants » avaient émigré ou s'étaient engagés dans l'armée nouvelle. Nous, nous étions ces rescapés de l'armée des vaincus de Bourguiba à la recherche d'un nouvel engagement. Et nous étions revenus pour ce faire aux capitales intellectuelles et spirituelles. Ce n'était pas à proprement parler une décision politique, c'était le résultat d'un sentiment confus d'aliénation, la recherche d'une vie meilleure, d'une insertion dans les grands centres d'appartenance. Une sorte de retour à l'authenticité. [...] L'Université tunisienne ayant été francisée, les diplômés de la Zitouna n'avaient d'autre choix que d'entrer à la faculté de théologie à Tunis. J'y ai suivi des cours un certain temps mais je voulais apprendre les sciences et (pour les arabophones) cela n'était pas possible à l'université. Les facultés du Machreq avaient, elles, des filières scientifiques arabisées, ce qui n'était pas le cas à Tunis. Le reste des Zitouniens est donc allé au Machreq, surtout au Caire, en Syrie et en Irak. La plupart sont sortis diplômés de sciences, surtout en sciences naturelles, mathématiques, polytechnique et médecine.

En Égypte, j'habitais à 'Agouza, chez des amis de mon village, étudiants comme moi. Nous sommes restés deux ou trois mois à nous démener pour obtenir une inscription à l'Université du Caire. La routine administrative, la bureaucratie... On a même manifesté devant le domicile de Nasser pour lui réclamer nos droits à l'inscription. Après des pressions intenses, nous avons réussi à nous inscrire. J'ai choisi la faculté d'Agriculture. Je me voyais alors passer ma vie à améliorer la condition des paysans de mon village et leurs méthodes de travail qui, à l'époque, étaient très arriérées. L'agriculture était dure, notamment à cause du manque d'eau. J'étais obligé d'aider mon père. J'en étais même arrivé une fois à interrompre mes études à la Zitouna. Mais cela $\mathrm{n}$ avait duré que quatre mois.

La société égyptienne? II m'est difficile d'en parler puisqu'à vrai dire je ne m'y étais pas intégré ; il ne m'a pas été donné de le faire. J'ai passé le plus clair de mon temps à essayer de m'installer: m'inscrire, faire toutes les démarches nécessaires pour la rentrée universitaire. Je n'ai visité qu'Alexandrie. À cette époque, je n'ai pas trouvé en Égypte ce que je rêvais d'y trouver. Je m'imaginais des Égyptiens enthousiastes, mobilisés derrière Nasser. J'ai vu un peuple davantage préoccupé de sa vie quotidienne. La mobilisation, les chants, les discours de ta radio, c'était une façade, un enthousiasme de commande auquel ne correspondait aucune réalité de la rue.

De toute façon, cette étape a très vite pris fin. Égypte et Tunisie ont rompu leurs relations diplomatiques. Le Caire avait soutenu Saleh Ben Youssef dans son conflit avec Bourguiba. Ce dernier avait fait appel à l'armée française et Ben Youssef à Nasser. C'est là une des raisons de la violente rancune de Bourguiba à l'égard de Nasser, du nationalisme arabe, du Machreq tout entier et de l'arabité. Une des raisons au moins. Par la suite, lorsque les relations bilatérales ont été restaurées, l'ambassade de Tunisie 
en Égypte s'est mise à nous rechercher pour nous signifier une interdiction de rester en Égypte. Et elle a fait pression sur l'administration égyptienne pour que nous soyons chassés de l'université. Après tous nos efforts pour obtenir une inscription, nous avons trouvé d'un seul coup nos noms barrés, et l'ambassade nous demandait de rentrer. Une partie d'entre nous l'a fait. L'armée victorieuse de Bourguiba poursuivait même les rescapés, même ceux qui s'étaient enfuis. Elle est donc parvenue à nous faire expulser d'Égypte. Il nous fallait accepter la capture et rentrer au pays ou fuir quelque part ailleurs. Alors je suis parti en Syrie. J'ai failli aller en .Albanie. De quoi est-ce que je vivais? J'avais emporté de l'argent de Tunisie. J'avais économisé à peu près 500 dinars tunisiens sur ma paie d'instituteur. Je dépensais petit à petit. En Égypte, je me contentais de foul et de ta'miyya, pour dépenser le moins possible.

\section{De l'arabisme à l'islamisme}

En Syrie par contre, nous nous sommes inscrits facilement. L'université était autonome par rapport à l'État. Et peu de temps après, le ministère de l'Education nous a accordé une bourse. Tous les étudiants tunisiens en recevaient. Pas importante mais suffisante. Damas n'est pas une grande ville comme Le Caire. Un étranger s'y retrouve, s'intègre plus facilement. La vie était très bon marché à l'époque. En fait, nous étions divisés en deux groupes : ceux qui avait été envoyés par le ministère tunisien de l'Education, et qui recevaient une bourse élevée et vivaient confortablement, et les autres, la majorité, qui étaient venus d'eux-mêmes et n'étaient pas boursiers. Lorsque Bourguiba fit son célèbre voyage au Machreq après qu'eut lieu la réconciliation, nous sommes allés le rencontrer à Beyrouth. Les non boursiers lui ont demandé l'attribution d'une bourse pour tous, qu'il leur a accordée de fait lors de son célèbre discours d'Ariha. On a donc tous bénéficié de la bourse tunisienne un certain temps.

Mais comme, dans ce fameux discours, il avait appelé à la reconnaissance d'Israël et au respect du découpage de 1948, l'opinion publique arabe, surtout syrienne, s'est révoltée. Il y a eu une émeute, l'ambassade de Tunisie a été brûlée, les relations entre la Syrie et la Tunisie ont été à nouveau rompues et les bourses suspendues. Un envoyé est venu nous demander de quitter ce pays dont le régime était hostile à la Tunisie et de rentrer chez nous. Certains l'ont fait, la majorité a refusé.

Quel était alors le climat politique chez les étudiants tunisiens ? [...] Les bourguibiens du contingent officiel nous poursuivaient. Il y avait bien sûr un consensus, un quasi consensus contre le régime tunisien. Tous étaient des victimes de cette guerre de Bourguiba contre l'islam et l'arabité. Un petit nombre de bourguibiens était toutefois en contact avec l'ambassade. La période libérale n'avait pas encore pris fin. Le socialisme et le ba'thisme n'avaient pas encore détruit la société syrienne. C'était en fait le début de l'expérience ba'thiste et le libéralisme économique permettait encore ce pays de prospérer. L'université jouissait alors de la liberté culturelle et politique. Tous les courants étaient représentés, dialogues et compétition politique se poursuivaient. Nous avons commencé à nous y intégrer. Le grand débat de l'époque opposait Ba'th aux nasséristes. La séparation entre l'Égypte et la Syrie n'était pas encore intervenue. La principale préoccupation des nasséristes, le centre de leur thématique, c'était la restauration de l'État unitaire. Compte tenu du background nassériste qui était le mien en Tunisie, je me suis assez naturellement et assez facilement intégré à leur courant. 
28 Entre le Ba'th et les nasséristes, il n'y avait pas de réelle différence. Ce fut là, d'ailleurs, une des raisons de ma métamorphose ultérieure: la découverte, que le débat mit rapidement en évidence, que nasséristes et ba'thistes se disputaient pour des raisons bien minces et bien futiles. Mais aussi dans le même temps, les progrès intellectuels que me permettaient mes lectures en philosophie jouèrent leur rôle. La philosophie conduit à se poser des questions sur tout, y compris sur le contenu effectif des mots: que voulait vraiment dire «arabité»? Que signifiait "unité » ou "socialisme»? L'ambiance de débat politique permanent nous poussait en fait à nous accrocher à des slogans bien davantage qu'à leur contenu. Le dialogue se déroulait entre nasséristes et ba'thistes, d'une part, et d'autre part, entre membres de la communauté tunisienne. Le groupe des Tunisiens était divisé entre ceux - la minorité - qui étaient restés bourguibistes, et la grande majorité, qui se partageait elle-même entre ba'thistes et nasséristes.

Le débat entre ces deux groupes était très intense. C'était la Ligue des étudiants tunisiens qui l'abritait. Il y avait des élections, des confrontations, une vie politique complète; on faisait sa campagne électorale, on défendait son programme. Les nasséristes étaient majoritaires. Les bourguibistes ne jouaient qu'un rôle marginal. Les islamistes ? Il n'y en avait pas. Il n'y en avait absolument pas. Pas un seul islamiste dans la Ligue. Pour autant que je me souvienne dans un groupe de 150 étudiants, il n'y en avait qu'un seul ou peut être deux qui faisaient la prière. On était en 1965. La prière ne faisait pas partie des représentations philosophiques ou politiques courantes. La lutte portait sur l'attitude vis-à-vis du régime tunisien.

30 Toutefois, ce débat politique n'avait pas seulement lieu entre Tunisiens. Il se déroulait également entre les arabistes d'un côté, toutes tendances confondues, et les islamistes. Les islamistes, à l'université syrienne, avaient une grande mosquée, où se donnait un prône le vendredi et ils avaient dans leurs rangs plusieurs personnalités célèbres. À l'époque, en Syrie, c'étaient soit des Frères Musulmans soit encore des membres du Parti de la Libération islamique. Il y avait aussi des individualités célèbres, officielles ou non, celles qui avaient le plus fort pouvoir d'attraction étant bien sûr les non officielles : enseignants à l'université, grands enseignants de la faculté de Chari'a. Donc le dialogue avait cours entre ces différents courants. Sur quoi portait-il ? Le fondement du débat à peu de choses près était politique : on débattait de l'attitude à avoir vis-à-vis des différents régimes, ou encore de la question palestinienne.

31 Le discours des Frères musulmans à l'époque se concentrait pour l'essentiel sur la condamnation de l'arabité, sur le fait qu'elle était laïque. Ils faisaient porter à la laïcité la responsabilité de la perte de la Palestine, celle de la corruption de la politique, des moeurs, de l'économie.

32 À l'opposé, le discours des nasséristes ou des ba'thistes contre les Frères musulmans consistait à dire qu'ils étaient un courant réactionnaire, lié au colonialisme, aux Américains. Ce progressisme était lui-même lié avec l'Europe de l'Est et traitait donc les Frères musulmans d'Américains, de valets de l'Amérique, ils étaient censés être contre le progrès, réactionnaires, etc.

33 L'Arabie Saoudite? Elle n'était pas présente à cette époque. Elle n'était pas encore présente sur le terrain du combat religieux. Elle ne l'est devenue qu'après la révolution du pétrole, lorsqu'elle s'est trouvée capable de faire imprimer des livres religieux proches de son point de vue et d'affirmer ainsi sa présence dans les milieux islamiques. 
À cette époque, les grandes sources idéologiques étaient plutôt l'Égypte, la Syrie et le Pakistan avec Abû 'Ala al-Mawdûdi.

Le Parti de la Libération? Il se concentrait sur la question du Califat. Le retour au Califat. Est-ce qu'il avait une assise sociale particulière? D'abord il ne représentait qu'une minorité. C'était un courant "salafì (fondamentaliste) qui prônait le retour aux sources premières de la religion. Ils avaient un cheikh de toute première importance, Nâsir al-Dîn al-Albani, considéré encore aujourd'hui dans le monde musulman comme un grand orateur. Il doit avoir aujourd'hui 80 ans et vit en Jordanie. $\mathrm{Au}$ nombre des personnalités emblématiques de la pensée religieuse, il y avait aussi Saïd Ramadan al-Boutî.

À cette époque, à l'intérieur du groupe des nasséristes, certains ont commencé à douter des présupposés du nationalisme arabe, qu'ils accusaient d'avoir un contenu non arabe, de n'être arabe que formellement. L'un des ouvrages très lus à cette époque était traduit du français : L'homme cet inconnu d'Alexis Carrel, un médecin. Ce n'était peutêtre pas un livre très connu en France, mais il était au centre de la littérature des islamistes de cette époque. Carrel a écrit aussi : Contemplations de l'homme, mais c'est son premier livre, traduit et plusieurs fois réédité, qui était le plus célèbre. Sayyid Qutb s'en est lui-même beaucoup servi pour critiquer la civilisation occidentale, pour mettre en évidence les malheurs de l'homme occidental - la dégradation de la famille et des rapports sociaux, ou celle des relations internationales - dus à sa civilisation. Il y avait aussi le livre de Springler, les écrits de Toynbee et aussi La chute de la civilisation, ouvrage d'un Anglais dont j'ai oublié le nom, ît bien d'autres encore. Comme l'une des composantes du débat consistait, à accuser le nationalisme arabe d'avoir un contenu occidental, non islamique, le fait de minimiser, de critiquer la civilisation occidentale faisait naturellement partie de l'argumentaire. C'est dans ce contexte que ces écrits, tout spécialement ceux d'Alexis Carrel, qui donnaient crédit à la position islamiste, prenaient leur valeur : la civilisation islamique détenait les traitements des maladies dont souffrait la civilisation occidentale. La modernisation ne nous avait pas transmis la science et la technologie mais seulement les maladies de l'Occident.

Un autre élément qui m'a préparé à accepter la critique du nationalisme arabe comme n'étant pas islamique mais occidental est mon voyage en Europe. Après avoir étudié un an à Damas, de janvier à juin 1965 à peu près, en première année de philosophie, j'ai quitté la Syrie et avec un groupe d'amis, nous sommes partis pour l'Europe. Turquie, Bulgarie, Yougoslavie, Autriche, Allemagne, France etc. Six mois en tout. [...] Je travaillais pour gagner de quoi vivre quelque temps, puis j'allais ailleurs. Je suis rentré enfin en Syrie au mois de janvier de l'année suivante. La plupart du temps, je logeais dans les auberges de jeunesse. Cela donnait l'occasion de discuter avec toutes sortes de jeunes. Eh bien! Je suis rentré en Syrie avec l'impression que la jeunesse occidentale vivait dans une sorte de confusion et cela m'a sans doute préparé à admettre cette critique de la civilisation occidentale (que véhiculait l'argumentaire des adversaires du nationalisme arabe). Ce voyage, et l'attitude de la jeunesse européenne côtoyée dans tes auberges de jeunesse, la rendaient plus acceptable.

Que représentait l'Union soviétique à ce moment-là ? Les apports les plus importants du discours islamiste, l'origine de sa force, étaient précisément le résumé très simple qu'il faisait de l'Occident, et le fait qu'il relativisait bon nombre de distinctions qui, pour d'autres étaient essentielles. Le débat entre capitalisme, socialisme, camp de l'Ouest, camp de l'Est n'avait plus cours : au fond, tout cela n'était qu'un seul et même 
Occident... Pour un jeune, le seul fait de casser ces classifications, sur le plan intellectuel, constituait un acte révolutionnaire. Il découvrait que les hommes vivaient dans I'erreur et qu'ils séparaient des choses qui, en dernière instance, étaient indissociables. Ce discours disait aux différents groupes en conflit, nasséristes, ba'thistes au libéraux: "Vous êtes tous dans le même sac. Vos références sont européennes et non pas nationalisteset en dernière instance, au niveau intellectuel, vous n'êtes que des agents de cet Occident ». Et cela aussi c'était une révolution. Parce que les nasséristes à l'époque se considéraient comme les représentants d'une grandiose révolution.

Les ba'thistes et les nasséristes se battaient pour des questions futiles, formelles. Nous leur disions : «Vous, en profondeur, vous n'êtes en fait que des agents de la civilisation occidentale. Vous n'avez aucune authenticité et c'est ce qui explique votre échec dans tous les domaines. Et vous échouerez dans la lutte contre Israël, vous échouerez. »

Et de fait, lorsque vint la guerre de 1967 - j'étais alors à Damas - les Jeunes ba'thistes en particulier considéraient qu'ils allaient pouvoir passer l'été sur les plages de Tel Aviv. C'était, pour ces jeunes nationalistes, nasséristes ou ba'thistes, une pure certitude. Ils croyaient dans le nassérisme et ils avaient des certitudes inébranlables. Le discours islamiste diffusait au contraire la certitude opposée. Celle que la défaite était inévitable car il n'était pas possible à une idéologie de cette sorte de conduire à la victoire. La défaite est venue bien sûr apporter un soutien essentiel au discours islamiste.

Pour ma part, la mutation (vers l'islamisme) s'était déjà opérée. Le moment le plus important, la clef la plus importante, fut une sorte d'explosion qui se produisiten moi. Elle me fit percevoir que l'arabité en laquelle nous croyions était en conflit avec l'islam. Et que, depuis très longtemps, j'étais dupe, si l'arabité pour laquelle j'étais prêt à mourir était antinomique avec l'islam qui m'avait nourri et dont j'étais fier.

41 Cette découverte a été difficile pour moi. Je suis rentré en tension avec la culture nassériste que j'avais jusqu'alors assumée. J'étais passé graduellement de membre à responsable d'une cellule qui comprenait 7 ou 8 personnes qui se réunissaient secrètement à des domiciles privés. J'ai entamé avec cette cellule un débat sur la relation entre l'arabité et la religion. Les membres de la cellule ne sont pas parvenus à me convaincre. Alors ils ont commencé à transmettre la question à la direction du Parti. Ils envoyaient régulièrement quelqu'un pour discuter avec moi. À la fin, j'étais d'accord pour rester dans le Parti mais à une condition: que la foi en Dieu y soit considérée comme une condition d'appartenance. Même à la manière des chrétiens. Ils prétextaient qu'il y avait des chrétiens parmi eux et je leur disais : « Même les chrétiens croient en Dieu, alors pourquoi ne pas faire de la foi un des éléments de l'appartenance au Parti? » Alors ils ont dit ; "Nous, on est un Parti qui n'instaure pas de relation entre la religion et la politique ", "la religion est à Dieu et la patrie à tous ", etc. J'ai été alors convaincu, lorsque la discussion en est arrivée là, que je m'étais longtemps trompé et que des années d'enthousiasme et de combat étaient fondées sur une illusion. Comme celui qui reniait sa famille, son éducation, sa civilisation et qui réalisait qu'il avait été prisonnier longtemps sans le savoir, croyant qu'il était libre alors qu'il vivait en prison et dans l'obscurité. C'est comme s'il s'était passé un renversement total dans ma vie. Je me souviens que la nuit du 15 juin, je n'ai pas pu fermer l'œil. J'étais très agité. Un peu avant l'aube, j'étais décidé à franchir le pas et à commencer à prier. J'ai fait une prière dont j'ignorais en fait les détails : j'avais oublié la culture religieuse.de mon enfance. Ma culture religieuse était une culture théorique et non pas une culture pratiquée, mise en 
oeuvre ou en actes. Et j'ai commencé à m'adonner au réapprentissage de la culture islamique, à lire et à faire l'inventaire du contenu des productions de l'islam. Les «marchandises » islamiques disponibles - disponibles au sein du courant islamiste étaient les livres de Sayyid Qutb, ceux de Muhammad Qutb, Abû 'Ala al-Mawdûdi, Muhammad Iqbal, Malek Bennabi et quelques écrits anciens de Abù-l-Hamid al-Ghazâli et Ibn Taymiyya. J'ai commencé à faire le tour des écoles religieuses de Damas. J'ai fait connaissance de l'école des Hadiths, du Fiqh, des différents groupes islamiques :

J'écoutais tout le monde, je voulais savoir ce que contenait l'univers de cette religion avec lequel je dialoguais de loin sans y être intégré [...]. »

\section{NOTES}

1. Cette démarche doit déboucher sur la publication prochaine (La Découverte, Paris) d'un ouvrage faisant suite à L'islamisme au Maghreb: la voix du Sud (Paris, Karthala, 1988) dont une version remaniée et actualisée est par ailleurs en cours de fabrication en langue arabe (Al-islam al-siyâsi : sawt al-janûb; Dar al-'Alam al-thâlith. Le Caire, 1992) et en langue anglaise (The islamic movement in the North of Africa, University of Texas Press, à paraître).

INDEX

Mots-clés : islamisme, nationalisme, politique, Tunisie

\section{AUTEUR}

FRANÇOIS BURGAT

CNRS-Cedej 